\title{
Clinical characteristics, laboratory outcome characteristics, comorbidities, and complications of related COVID-19 deceased: a systematic review and meta-analysis
}

\author{
Peishan Qiu ${ }^{1,2} \cdot$ Yunjiao Zhou ${ }^{1,2} \cdot$ Fan Wang $^{1,2} \cdot$ Haizhou Wang $^{1,2} \cdot$ Meng Zhang $^{1,2} \cdot$ Xingfei Pan $^{3} \cdot$ Qiu Zhao $^{1,2} \cdot$ \\ Jing Liu ${ }^{1,2}$ (D)
}

Received: 9 May 2020 / Accepted: 17 July 2020 / Published online: 30 July 2020

(c) Springer Nature Switzerland AG 2020

\begin{abstract}
Background At present, novel coronavirus disease 2019 (COVID-19) has become a serious global public health problem. The current meta-analysis aimed to find risk factors for the COVID-19-related death, helping to enhance the efficacy and reduce the mortality of COVID-19.

Methods We searched PubMed, Embase, medRxiv, and Cochrane Library for articles published between January 1, 2020, and April 13, 2020. We statistically analyzed the risk factors of the COVID-19 deceased with meta-analysis.

Results A total of 2401 patients in 15 articles were included in this study. Meta-analysis showed that $66.6 \%$ of COVID-19 deceased were male, with a median age of 69.9 years. Common symptoms of deceased included fever (70.6-100\%), dyspnea (38.89-85.7\%), cough (22.4-78\%), and fatigue (22-61.9\%). The incidence of hypertension, chronic cardiovascular disease, diabetes, and chronic cerebrovascular disease among the COVID-19 deceased were 38.56\% (95\% confidence interval (CI) $25.84 \sim 52.12 \%$ ), $17.54 \%$ (95\% CI 13.38 21.69\%), 22.2\% (95\% CI 19.30 25.10\%), and 15.58\% (95\% CI 10.05 21.12\%), respectively. Compared with the surviving COVID-19 patients, the deceased had lower platelet levels (mean difference $(\mathrm{MD})=-39.35,95 \% \mathrm{CI}-55.78 \sim-22.93)$ and higher C-reactive protein (CRP) $(\mathrm{MD}=80.85,95 \% \mathrm{CI} 62.53 \sim 99.18)$ and lactate dehydrogenase $(\mathrm{LDH})(\mathrm{MD}=246.65,95 \% \mathrm{CI} 157.43 \sim 335.88)$ at admission. The most common complications of the deceased were acute respiratory distress syndrome (ARDS) $(\mathrm{OR}=100.36,95 \% \mathrm{CI} 64.44 \sim 156.32)$ and shock $(\mathrm{OR}=96.60$, $95 \%$ CI 23.80 392.14).

Conclusion Most of the COVID-19 deceased were elderly males. Fever, dyspnea, dry cough, fatigue, hypertension, chronic cardiovascular and cerebrovascular disease, diabetes, and laboratory examinations showed low levels of platelet content, increased CRP and LDH were associated with the risk of dying. ARDS and shock were risk factors for death in COVID-19 patients.
\end{abstract}

Keywords Death · COVID-19 - Severe acute respiratory syndrome coronavirus $2 \cdot$ Meta-analysis

Peishan Qiu, Yunjiao Zhou and Fan Wang contributed equally to this work.

Xingfei Pan

panxf0125@163.com

$\checkmark$ Qiu Zhao

qiuzhao@whu.edu.cn

$\triangle$ Jing Liu

liujing_GI@whu.edu.cn

Extended author information available on the last page of the article

\section{Introduction}

Since the outbreak of the new coronavirus (severe acute respiratory syndrome coronavirus 2 [SARS-CoV-2], formerly known as 2019-nCoV) in December 2019 in Wuhan, Hubei Province, China [1-3], confirmed cases have appeared in countries around the world. Genome sequencing and phylogenetic analysis show that SARS-CoV-2 is a new type of human-infected $\beta$-coronavirus. Bats are presumed to be the original host of this zoonotic virus, but there is no evidence that there is an intermediate host that promotes human infection with the virus [4]. According to the Coronavirus disease 2019 (COVID-19) Situation Report-84 [5] released by 
the World Health Organization (WHO) on April 13, there were 1,773,084 confirmed cases of COVID-19 and 111,652 deaths worldwide. Patients infected with SARS-CoV-2 were prone to death when they develop severe pneumonia, pulmonary edema, ARDS, or multiple organ failure (such as shock, acute heart injury, and acute kidney injury) [6]. The increasing number of deaths forces us to find out the clinical characteristics of patients at risk of dying, so that clinical workers can take corresponding rescue measures in the early stage to reduce the occurrence of death.

At present, more and more retrospective studies have reported the clinical information of COVID-19 dead patients. Here we will provide systematic assessment and details, not only to assess the disease coexistence of all dead patients, the results of laboratory examinations, but also to compare the potential disease risks between survival and death patients. These results may assist management in developing prevention policies to address COVID-19 and its key results.

\section{Methods}

\section{Search strategy and selection criteria}

Literature search in the PubMed, Embase, medRxiv (https:// www.medrxiv.org/), and Cochrane Library from January 1, 2020 to April 13, 2020, using a combination of the following keywords: "SARS-CoV-2" or "2019-nCoV" or "COVID19 " or "new coronavirus" or "Wuhan Coronavirus" and "die" or "death" or "fatality" or "mortality" or "decease" or "survive" or "survivors". Restrict publication language to English. In addition, to ensure the comprehensiveness and accuracy of the research, we also consulted the references of the included literature. This work was independently completed by two authors (Peishan Qiu and Yunjiao Zhou). Disagreements were resolved by the third investigator (Fan Wang). All the search results were evaluated according to the preferred reporting items for systematic reviews and meta-analyses (PRISMA) statement.

\section{Inclusion and exclusion criteria}

The inclusion criteria of the meta-analysis were as follows: (1) the cases in each study were patients diagnosed with COVID-19, (2) involving the death group or non-survivor group and the survivor group, (3) at least one outcome reported among demographical characteristics, comorbidities, and clinical manifestations of COVID-19 deceased. Studies such as (a) repetitive publications, (b) reviews, editorials, case reports, letters, and family-based studies, and (c) studies with fewer than five cases were excluded.

\section{Data extraction and paper quality evaluation}

Data extraction and literature quality evaluation were completed by two investigators (Peishan Qiu and Yunjiao Zhou) independently. Disagreements were resolved with a third investigator (Fan Wang) or by consensus. All included papers were evaluated using the Newcastle-Ottawa scale [7]. The following variables were extracted: the authors, the baseline details, clinical manifestations, laboratory examinations, and clinical outcomes.

\section{Statistical analysis}

Single-arm meta-analysis was performed using R 3.6.3 software. Review Manager 5.3 (RevMan version 5.3) software was used to compare the clinical characteristics and laboratory results of COVID-19 deceased and surviving patients. The odds ratio (OR, 95\% confidence intervals $(\mathrm{CI})$ ) was used to describe the ratio of the probability of the complications occurring in dead patients vs. survivor. Mean difference (MD, 95\% CI) was used to describe the differences in the mean of laboratory examinations between dead and surviving COVID-19 patients. Heterogeneity among the studies was assessed by the Cochran Chi-square and $I^{2}$ test. When $I^{2}<50 \%$, a fixed-effect model was used, otherwise a randomeffect model was selected. When there was statistical heterogeneity among the results, a further sensitivity analysis was performed to determine the source of heterogeneity. The publication bias was assessed using a funnel plot and quantified using Egger's linear regression test. $P<0.05$ was statistically significant (two sided).

\section{Results}

\section{Characteristics of included studies}

As shown in Fig. 1, the total number of records initially determined based on the search strategy was 2679. After deleting 462 duplicates, we deleted another 2134 articles by reading the title and abstract of the article. We eliminated 68 articles by reading the full-text articles of the remaining 83 studies, 35 of which had no clinical data of death, 30 of which did not provide clinical data related to our study, and 3 of which included less than 5 cases. Finally, there were 15 articles included in our meta-analysis [8-22]. 


\section{Clinical manifestation of dead patients with COVID-19}

The clinical data study included 15 studies [8-22] with a total of 2401 cases, including 904 deceased. The epidemiology, baseline characteristics, and clinical symptoms of all deaths included are summarized in Table 1. The median age of COVID19 deaths was 69.9 years, with $66.6 \%$ males. The main clinical symptoms of COVID19 dead patients on admission were fever (70.6-100\%), dyspnea (38.89-85.7\%), cough (22.4-78\%), fatigue (22-61.9\%), expectoration (22.2-57.1\%), myalgia (2.78-33.3\%), diarrhea (4-38.1\%), anorexia (23.4-56.5\%). Other rare symptoms included chest urgency (2.4-49\%), headache (4.7-23.8\%), sore throat (2.4-4.7\%), hemoptysis (4-5.56\%), nausea and vomiting (4-4.7\%), abdominal pain (3.5-5\%), and dizziness (9\%).

\section{Prevalence of underlying diseases in COVID-19 deaths}

Through the current meta-analysis, we found that the prevalence rate of underlying diseases among COVID-19 dead patients was $72.21 \%$ (95\% CI $62.85 \sim 79.96 \%$ ).
Fig. 1 Flow diagram of the number of studies screened and included in the meta-analysis

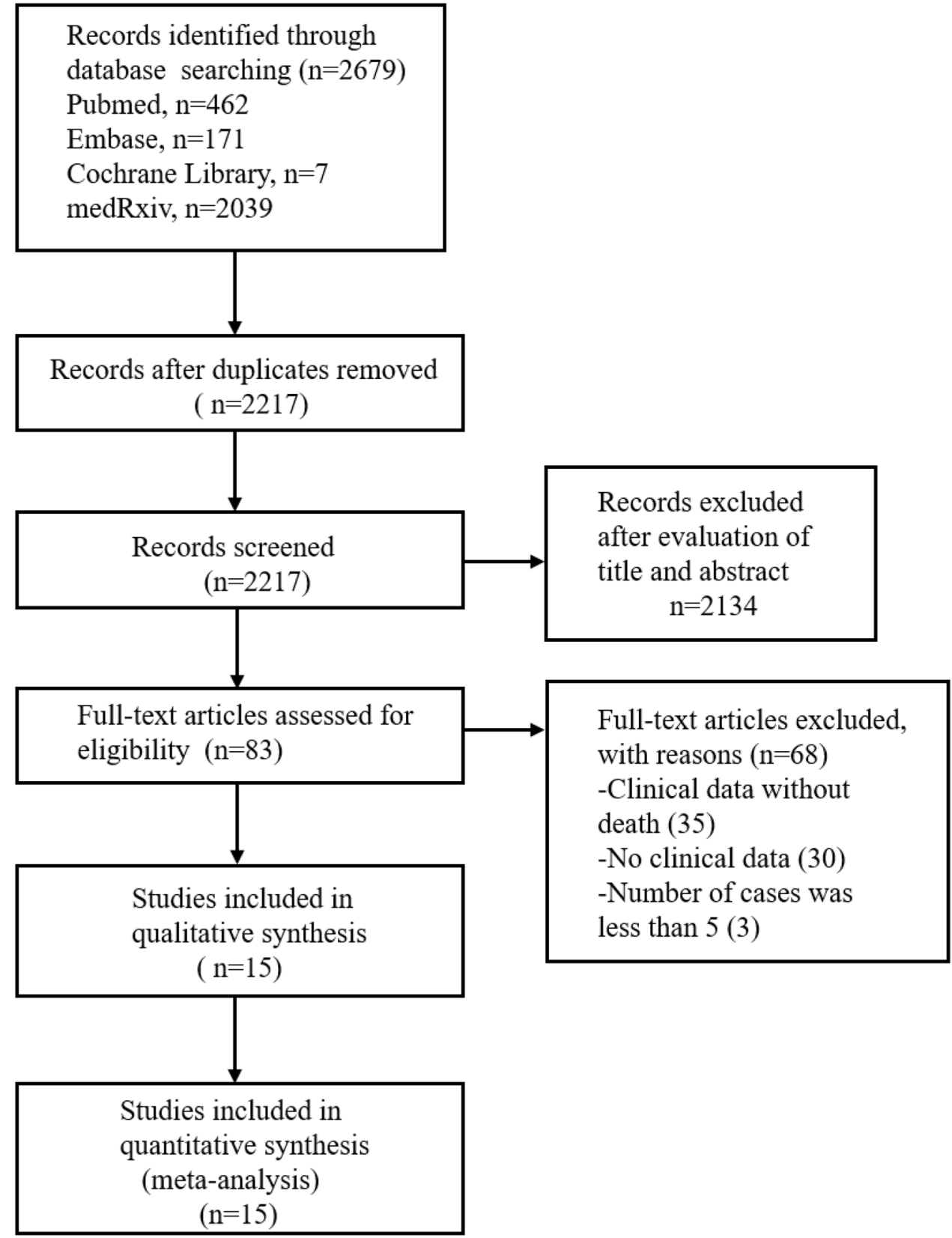




\section{Hypertension}

In a comprehensive analysis of 14 studies [8-17, 19-22], the pooled prevalence of hypertension in the COVID-19 deceased was estimated as $38.56 \%$ (95\% CI 25.84 52.12\%) (Fig. 2a). According to the results of the $I^{2}$ index (93\%) and the Chi-square test, there was high and significant heterogeneity between the studies $(P<0.01)$, also no publication bias was present according to Eggers's test $(t=0.083162$, $P=0.9351)$.

\section{Chronic cardiovascular disease}

To estimate the pooled prevalence of the cardiovascular disease in COVID-19 deceased, 14 studies were evaluated [8-17, 19-22]. The incidence was $23.48 \%$ (95\% CI $16.75 \sim 30.20 \%$ ), with high and significant heterogeneity $\left(I^{2}=83 \%\right)$. There was publication bias by Eggers's test $(t=3.2692, P=0.006713)$. Through sensitivity analysis, it was found that the heterogeneity significantly decreased $\left(I^{2}=52 \%\right)$ after the exclusion of data from the "Korean" group [14] and the "Du RH" group [11]. The prevalence of meta-analysis after excluding studies affecting heterogeneity was $17.54 \%$ (95\% CI 13.38 21.69\%) (Fig. 2b), and there was no publication bias according to Eggers's test $(t=3.1061, P=0.01113)$.

\section{Diabetes}

The pooled prevalence of diabetes in COVID-19 death patients was estimated to be $22.2 \%$ (95\% CI 19.30 25.10\%). The heterogeneity of the study was low $\left(I^{2}=28.4 \%\right.$, $P=0.1519$ ) (Fig. 2c). A fixed model was used to perform a meta-analysis of data from 14 studies [8-17, 19-22].

\section{Chronic cerebrovascular disease}

After the combination of 12 studies [8, 9, 11-17, 19-21], the random-effects model was used to calculate the prevalence of the cerebrovascular disease in COVID-19 deceased was $15.58 \%$ (95\% CI $10.05 \sim 21.12 \%, I^{2}=77.7 \%, P<0.01$ ) (Fig. 2d). The heterogeneity of the study was high, using a random-effects model. The sensitivity analysis was relatively stable.

\section{Other comorbidities}

The results of the meta-analysis showed that the incidence of chronic lung disease in COVID-19 deceased was 14.2\% (95\% CI $11.27 \sim 17.21 \%)$. The prevalence of tumors $(4.28 \%$, 95\% CI $2.21 \sim 6.99 \%)$, chronic kidney disease $(4.17 \%, 95 \%$ CI $2.6 \sim 5.74 \%)$, and chronic liver disease $(2.86 \%, 95 \% \mathrm{CI}$
1.6 4.48\%) were slightly increased in COVID-19 deaths. There was no publication bias by Egger's test.

\section{Characteristics of laboratory examinations of COVID-19 death patients}

The purpose of this study was to explore the changes in laboratory indexes related to the poor prognosis of COVID19 by comparing the laboratory examinations of the final dead patients with COVID-19 and the surviving patients (Table 2).

Through meta-analysis, we found that compared with patients who were also infected with SARS-CoV-2 but whose final outcome was alive, laboratory examinations showed a slightly higher leucocytes count $(\mathrm{MD}=3.77$, 95\% CI $2.90 \sim 4.64)$, neutrophil count increased slightly ( $\mathrm{MD}=4.57,95 \%$ CI 2.92 6.23), lymphocyte count decreased $(\mathrm{MD}=-0.37,95 \% \mathrm{CI}-0.47 \sim 0.26)$, platelets count decreased significantly $(\mathrm{MD}=-39.35,95 \% \mathrm{CI}$ $-55.78 \sim-22.93$ ), D-dimer slightly increased $(\mathrm{MD}=2.96$, 95\% CI $1.93 \sim 3.98$ ), CRP increased significantly $(\mathrm{MD}=80.85,95 \%$ CI 62.53 99.18), alanine transaminase (ALT) slightly increased (MD $=5.07,95 \%$ CI $0.26 \sim 9.88$ ), aspartate transaminase (AST) significantly increased $(\mathrm{MD}=14.26,95 \% \mathrm{CI} 11.70 \sim 16.81)$, blood urea nitrogen (BUN) slightly increased (MD $=4.00,95 \%$ CI $3.33 \sim 4.67$ ), serum creatinine (Scr) was significantly increased $(\mathrm{MD}=21.20$, 95\% CI 16.79-25.61), and LDH was abnormally increased ( $\mathrm{MD}=246.65,95 \%$ CI $157.43 \sim 335.88)$, creatine kinase $(\mathrm{CK})$ increased $(\mathrm{MD}=48.98,95 \% \mathrm{CI}$ 3.53 94.43), plasma albumin decreased ( $\mathrm{MD}=-4.00,95 \%$ CI $-5.17 \sim-2.83$ ), total bilirubin (Tbil) slightly increased $(\mathrm{MD}=3.92,95 \%$ CI $1.94 \sim 5.91)$, interleukin-6 (IL-6) significantly increased ( $\mathrm{MD}=27.57,95 \%$ CI $17.45 \sim 37.69)$, prothrombin time (PT) slightly increased (MD $=1.13,95 \%$ CI $0.67 \sim 1.60$ ). However, there was no significant difference in hemoglobin, procalcitonin (PCT), and activated partial thromboplastin time (APTT) between the two groups.

\section{Occurrence of complications of COVID-19 death patients}

We compared the incidence of complications between dead and surviving patients after the SARS-CoV-2 infection. A total of eight studies $[8-10,17,19-22]$ were included in the meta-analysis.

\section{Acute respiratory distress syndrome (ARDS)}

A total of eight studies were included [8-10, 17, 19-22]. The results showed that compared with the patients who survived COVID-19, the most common complication of the patients who eventually died was ARDS (OR $=75.05,95 \%$ 


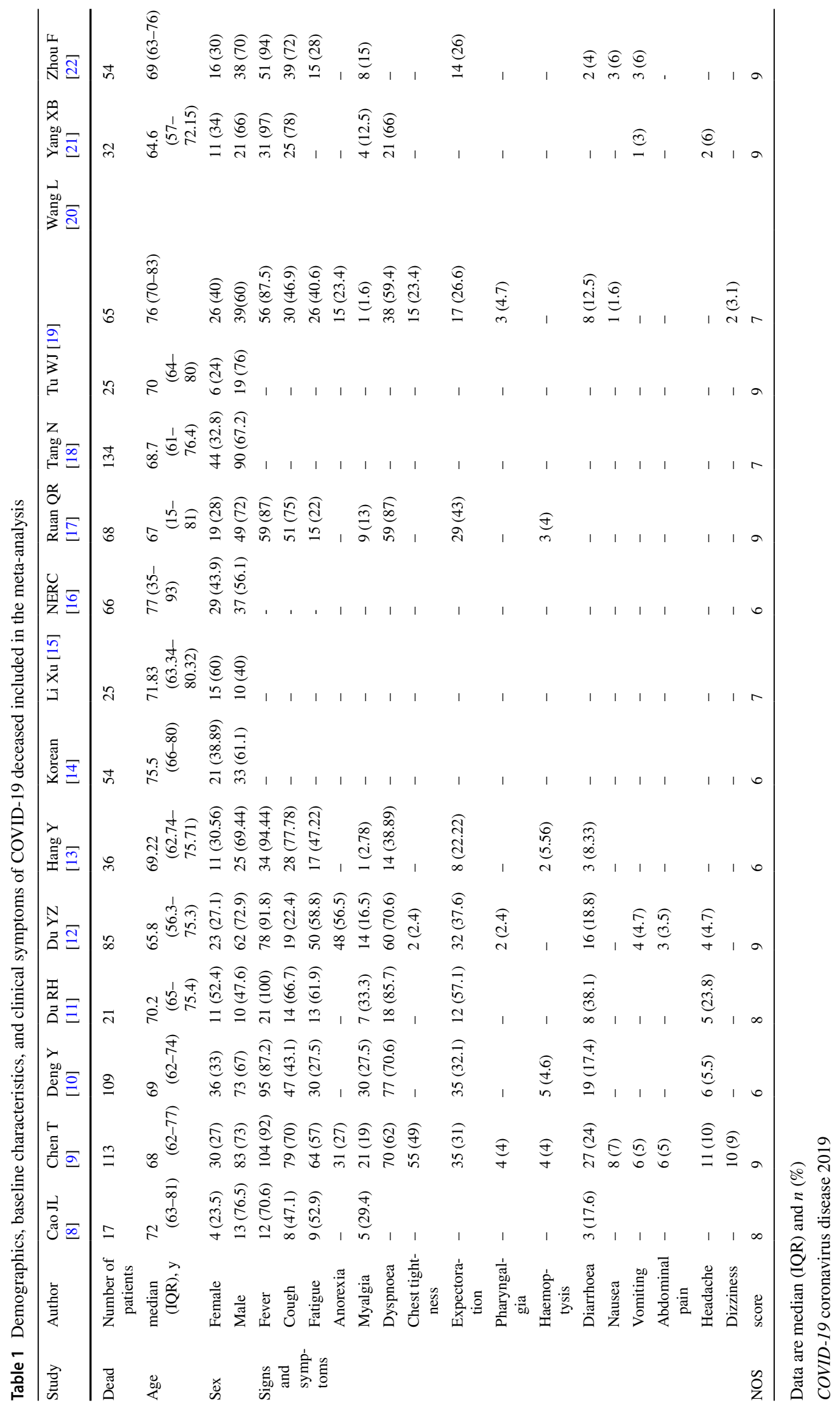


CI 33.35 168.86, $P<0.00001)$. The heterogeneity of the study was high $\left(I^{2}=70 \%\right)$, and Egger's test showed publication bias $(P=0.001)$. The sensitivity analysis showed that the heterogeneity of the study was significantly reduced $\left(I^{2}=0 \%\right)$ after excluding the data of the "Yang XB" group [21]. Meta-analysis showed that ARDS was a risk factor for death in COVID-19 patients (OR $=100.36,95 \%$ CI 64.44 156.32, $P<0.00001$ ) (Fig. 3a). The Egger's test showed that there was no publication bias $(P=0.61)$.

\section{Shock}

A total of six studies were included [8-10, 19, 20, 22]. We found that dead patients were more likely to develop shock $\left(\mathrm{OR}=51.55,95 \%\right.$ CI $8.32 \sim 319.28, P<0.0001, I^{2}=80 \%$, $P=0.001$ ) than COVID-19 survivors, but the heterogeneity of the study was high. The sensitivity analysis showed that the heterogeneity of this study decreased $\left(I^{2}=52 \%, P=0.08\right)$ after excluding the "Wang L" group [20]. Therefore, the shock was a risk factor for death in patients COVID-19 $(\mathrm{OR}=96.60,95 \%$ CI 23.80 392.14, $P<0.00001)$ (Fig. 3b).

\section{Other complications}

Compared with the surviving patients, the incidence of infection $(\mathrm{OR}=30.25,95 \%$ CI 6.86 133.39, $P<0.00001$, $\left.I^{2}=82 \%, P<0.0001\right)$, acute renal failure (AKI) $(\mathrm{OR}=28.11$, 95\% CI 10.73 73.66, $P<0.00001, I^{2}=69 \%, P=0.002$ ), acute heart injury $(\mathrm{OR}=27.73,95 \%$ CI $11.00 \sim 69.89$, $\left.P<0.00001, I^{2}=78 \%, P=0.0002\right)$ and acute liver injury $\left(\mathrm{OR}=3.15,95 \%\right.$ CI $1.29 \sim 7.70, P=0.01, I^{2}=75 \%$, $P=0.003)$ increased in patients with COVID-19 death.

\section{Discussion}

This meta-analysis included data from 15 laboratory-confirmed COVID-19 studies. Through our analysis, we found that $66.6 \%$ (602/904) of the dead patients were male, and the median age was 69.9 years old, in line with Huang et al. [3]. Common symptoms of COVID-19 deaths include fever, dyspnea, cough, and fatigue. Hypertension, chronic cardiovascular disease, diabetes, and chronic cerebrovascular disease are common comorbidities among COVID-19 deceased. COVID-19 patients with complications such as ARDS and shock are more likely to die. Laboratory examinations showed low levels of platelet content, increased CRP and LDH were associated with death in patients with COVID-19.

The elderly and men were the susceptible factors of COVID-19. For the high mortality rate of COVID-19 among the elderly, we believe that it may be related to the high prevalence of comorbidities among the elderly. Studies of the Middle East respiratory syndrome coronavirus (MERS$\mathrm{CoV}$ ) and SARS-CoV also found more infections in men than women $[23,24]$. On the one hand, it is generally believed that women have stronger innate and adaptive immune responses than men [25], which may be due to the protection of $X$ chromosomes and sex hormones [26]. For this reason, women are less likely to affect a variety of bacteria and viruses than men. On the other hand, men tend to have more bad habits than women (such as smoking, drinking, more underlying diseases), which may also be the reason why men with COVID-19 are more likely to die.

Chronic diseases have become a global economic burden [27]. Our study revealed that the prevalence of chronic underlying diseases in dead patients with COVID-19 was $72.21 \%$ (95\% CI 62.85-79.96\%). Compared to subjects with no comorbidities, severe pandemic influenza cases were significantly increased in patients with obesity, cardiovascular diseases, and hypertension [28]. The study of the data extracted from the severe MERS-CoV cases suggested that diabetes and hypertension were equally prevalent in approximately $50 \%$ of the patients [23]. The state of hyperglycemia and insulin resistance in diabetic patients would weaken the synthesis of pro-inflammatory cytokines such as interferon- $\gamma$ and interleukin and their downstream acute phase reactants [29], which makes diabetic patients more susceptible to SARS-CoV-2. Simultaneously, viral infection may cause sharp fluctuations in blood glucose levels in patients with diabetes, which will adversely affect the rehabilitation of patients. The angiotensin-converting enzyme 2 (ACE2) has been proved to be the receptor for SARS-CoV-2 to enter the host cells [6]. A study analyzed the lung transcriptome samples of more than 700 patients with comorbidities associated with severe COVID-19 and found that ACE2 was highly expressed in these patients compared to the control individuals [30]. Hence, patients with comorbidities may have higher chances of developing severe COVID-19. Lippi et al. believed that hypertension was a clinical predictor of COVID-19 severity in elderly patients [31]. Our research showed that the incidence of hypertension in patients with COVID-19 death was $38.56 \%$ (95\% CI $25.84 \sim 52.12 \%$ ). Tikellis et al. found that the expression of ACE2 in patients with hypertension might be decreased [32], increasing the level of angiotensin II, which would promote the development of ARDS [33].

In a study of the pathophysiology of ARDS, increased inflammation, and lung injury in rat models were associated with increased age, which was caused by an imbalance in the lung renin-angiotensin system (RAS) [34]. We observed that patients who died were more likely to have ARDS than those who survived with COVID-19 (OR $=75.05,95 \%$ CI 33.35-168.86). And as mentioned earlier, the elderly were the majority of the dead. ACE2 can antagonize the activation of the classical RAS and mediate vasodilation, 
A

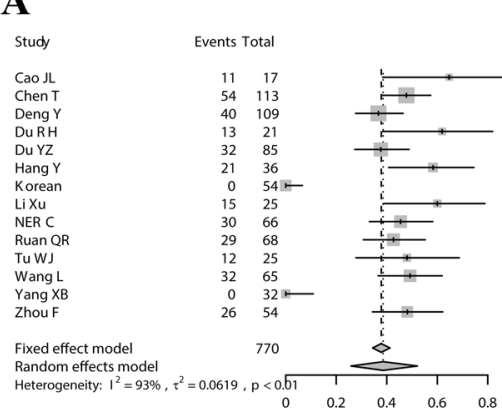

C

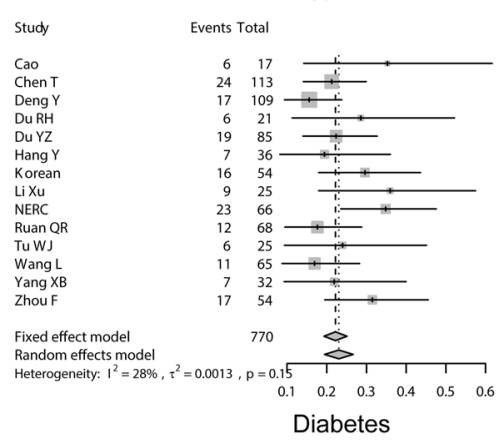

Diabetes
B
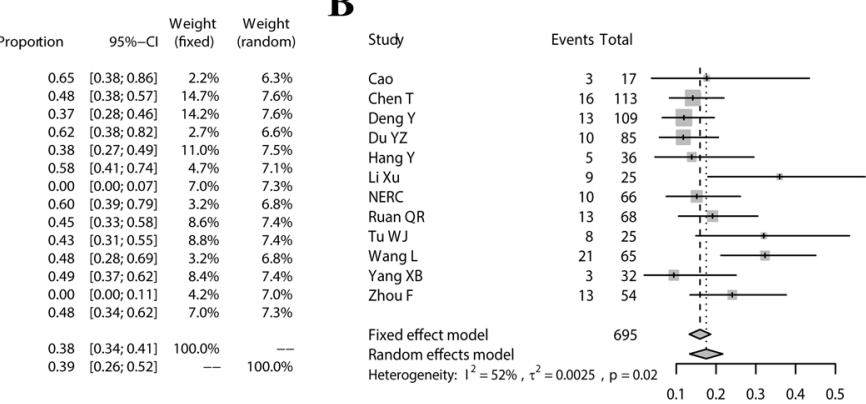

Proportion $\quad 95 \%-\mathrm{Cl} \begin{array}{r}\text { Weight } \\ \text { (fixed) }\end{array} \quad \begin{array}{r}\text { Weight } \\ \text { (random) }\end{array}$

$\begin{array}{rrrr}0.18 & {[0.04 ; 0.43]} & 2.2 \% & 4.1 \% \\ 0.14 & {[0.08 ; 0.22]} & 17.4 \% & 12.4 \%\end{array}$

$\begin{array}{llll}0.14 & {[0.08 ; 0.22]} & 17.4 \% & 12.4 \% \\ 0.12 & {[0.07: 0.20]} & 19.4 \% & 128 \%\end{array}$

$0.12[0.06 ; 0.21] \quad 15.3 \% \quad 12.0 \%$

$0.14[0.05 ; 0.29] \quad 5.6 \% \quad 7.7 \%$

$0.36[0.18 ; 0.57] \quad 2.0 \% \quad 3.8 \%$

$\begin{array}{rrrr}0.15 & {[0.08 ; 0.26]} & 9.6 \% & 10.0 \% \\ 0.19 & {[0.11 ; 0.30]} & 8.2 \% & 93 \%\end{array}$

$\begin{array}{llll}0.19 & {[0.11 ; 0.30]} & 8.2 \% & 9.3 \% \\ 0.32 & {[0.15 ; 0.54]} & 2.1 \% & 4.0 \% \\ 0.32 & {[0.21 ; 0.5]} & 5.6 \% & \end{array}$

$\begin{array}{llll}0.32 & {[0.15 ; 0.54]} & 2.1 \% & 4.0 \% \\ 0.32 & {[0.21 ; 0.45]} & 5.6 \% & 7.6 \%\end{array}$

$0.09[0.02 ; 0.25] \quad 7.0 \% \quad 8.7 \%$

$0.24[0.13 ; 0.38] \quad 5.5 \% \quad 7.6 \%$

$\begin{array}{lrrr}0.16 & {[0.13 ; 0.19]} & 100.0 \% & -- \\ 0.18 & {[0.13 ; 0.22]} & -- & 100.0 \%\end{array}$

D

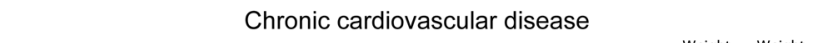

Propotion $\quad 95 \%-\mathrm{Cl}$ Weight $\begin{array}{r}\text { Weight } \\ \text { (fixed) }\end{array}$

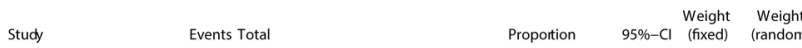

Study Events Total $\quad$ Proporon-Cl (fixed) (random

$\begin{array}{lrrrrrr}\text { Cao JL } & 3 & 17 & 0.18 & {[0.04 ; 0.43]} & 1.5 \% & 5.4 \% \\ \text { Chen T } & 4 & 113 & 0.04 & {[0.01 ; 0.09]} & 41.8 \% & 12.2 \%\end{array}$

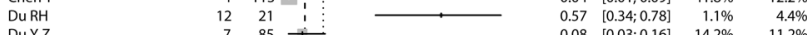

$\begin{array}{lllllll}\text { DuYZ } & 7 & 85 & 0.08 & {[0.03 ; 0.16]} & 14.2 \% & 11.2 \% \\ \text { Hang } & 8 & 36 & 0.22 & {[0.10 ; 0.39]} & 2.6 \% & 7.2 \%\end{array}$

\begin{tabular}{lrrrrrrr} 
Hang $Y$ & 86 & 0.22 & {$[0.10,0.39]$} & $2.6 \%$ & $7.2 \%$ \\
Korean & 10 & 54 & 0.19 & $0.09 ; 0.31]$ & $4.5 \%$ & $8.8 \%$ \\
LiXu & 3 & 25 & & 0.12 & {$[0.03 ; 0.31]$} & $3.0 \%$ & $7.6 \%$ \\
\hline
\end{tabular}

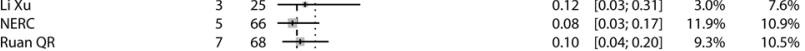

$\begin{array}{lcccccc}\text { Ruan QR } & 7 & 68 & 0.10 & {[0.04 ; 0.20]} & 9.3 \% & 10.5 \% \\ \text { TuWJ } & 8 & 25 & 0.32 & {[0.15 ; 0.54]} & 1.5 \% & 5.3 \%\end{array}$

$\begin{array}{lrr:rrrrr}\text { Wang } L & 10 & 65 & - & 0.15 & {[0.08 ; 0.26]} & 6.3 \% & 9.7 \% \\ \text { Yang XB } & 7 & 32 & -0.09 ; 0.40] & 2.4 \% & 6.9 \%\end{array}$

Random effects model

$607 \diamond$

$0.22[0.09 ; 0.40] \quad 2.4 \% \quad 6.9 \%$

$\begin{array}{llrr}0.09 & {[0.07 ; 0.11]} & 100.0 \% & - \\ 0.16 & {[0.10 ; 0.21]} & - & 100.0 \%\end{array}$

Chronic cerebrovascular disease

Fig. 2 Meta-analysis of the prevalence of comorbidities in COVID-19 deaths. a Hypertension; b chronic cardiovascular disease; c diabetes; d chronic cerebrovascular disease

Table 2 Comparison of laboratory results between COVID-19 deceased and surviving patients

\begin{tabular}{|c|c|c|c|c|c|c|}
\hline & \multirow{2}{*}{$\begin{array}{l}\text { No. of stud- } \\
\text { ies }\end{array}$} & \multirow[t]{2}{*}{ MD $(95 \% \mathrm{CI})$} & \multirow[t]{2}{*}{$P$ value } & \multicolumn{2}{|c|}{ Heterogeneity } & \multirow[t]{2}{*}{ Model used } \\
\hline & & & & $\overline{I^{2}}$ & $P_{h}$ & \\
\hline White blood cell count, $\times 10^{9} / \mathrm{L}$ & 7 & $3.77(2.90,4.64)$ & $<0.00001$ & $60 \%$ & 0.02 & Random \\
\hline Neutrophil count, $\times 10^{9} / \mathrm{L}$ & 3 & $4.57(2.92,6.23)$ & $<0.00001$ & $71 \%$ & 0.03 & Random \\
\hline Lymphocyte count, $\times 10^{9} / \mathrm{L}$ & 8 & $-0.37(-0.47,-0.26)$ & $<0.00001$ & $80 \%$ & $<0.0001$ & Random \\
\hline Haemoglobin, g/L & 5 & $-0.30(-2.84,2.24)$ & 0.82 & 0 & 0.93 & Fixed \\
\hline Platelets, $\times 10^{9} / \mathrm{L}$ & 6 & $-39.35(-55.78,-22.93)$ & $<0.00001$ & $65 \%$ & 0.01 & Random \\
\hline D-dimer, mg/L & 6 & $2.96(1.93,3.98)$ & $<0.00001$ & $0 \%$ & 0.61 & Fixed \\
\hline $\mathrm{CRP}, \mathrm{mg} / \mathrm{L}$ & 6 & $80.85(62.53,99.18)$ & $<0.00001$ & $73 \%$ & 0.002 & Random \\
\hline PCT, ng/mL & 4 & $0.15(-0.04,0.34)$ & 0.12 & $86 \%$ & 0.0008 & Random \\
\hline ALT, U/L & 6 & $5.07(0.26,9.88)$ & 0.04 & $74 \%$ & 0.002 & Random \\
\hline AST, U/L & 5 & $14.26(11.70,16.81)$ & $<0.00001$ & $48 \%$ & 0.11 & Fixed \\
\hline $\mathrm{BUN}, \mathrm{mmol} / \mathrm{L}$ & 3 & $4.00(3.33,4.67)$ & $<0.00001$ & $0 \%$ & 0.52 & Fixed \\
\hline $\mathrm{Scr}, \mu \mathrm{mol} / \mathrm{L}$ & 6 & $21.20(16.79,25.61)$ & $<0.00001$ & $13 \%$ & 0.33 & Fixed \\
\hline LDH, U/L & 4 & $246.65(157.43,335.88)$ & $<0.00001$ & $85 \%$ & 0.0002 & Random \\
\hline CK, U/L & 4 & $48.98(3.53,94.43)$ & 0.03 & $78 \%$ & 0.004 & Random \\
\hline Alb, g/L & 4 & $-4.00(-5.17,-2.83)$ & $<0.00001$ & $0 \%$ & 0.77 & Fixed \\
\hline Tbil, mmol/L & 4 & $3.92(1.94,5.91)$ & 0.0001 & $59 \%$ & 0.06 & Random \\
\hline IL-6, pg/mL & 5 & $27.57(17.45,37.69)$ & $<0.00001$ & $96 \%$ & $<0.00001$ & Random \\
\hline PT, seconds & 6 & $1.13(0.67,1.60)$ & $<0.00001$ & $60 \%$ & 0.03 & Random \\
\hline APTT, seconds & 3 & $0.64(-0.17,1.44)$ & 0.12 & $22 \%$ & 0.28 & Fixed \\
\hline
\end{tabular}

COVID-19 Coronavirus disease 2019, MD mean difference, $C I$ confidence interval, $C R P$ C-reactive protein, $P C T$ procalcitonin, $A L T$ alanine transaminase, $A S T$ aspartate transaminase, $B U N$ blood urea nitrogen, $S c r$ serum creatinine, $L D H$ lactate dehydrogenase, $C K$ creatine kinase, $A l b$ albumin, Tbil total bilirubin, $I L-6$ interleukin-6, PT prothrombin time, APTT activated partial thromboplastin time

$P \leq 0.05$ was considered to be statistically significant 
Fig. 3 Meta-analysis of the comparison of complications between COVID-19 dead patients and surviving patients. a Acute respiratory distress syndrome (ARDS); b shock
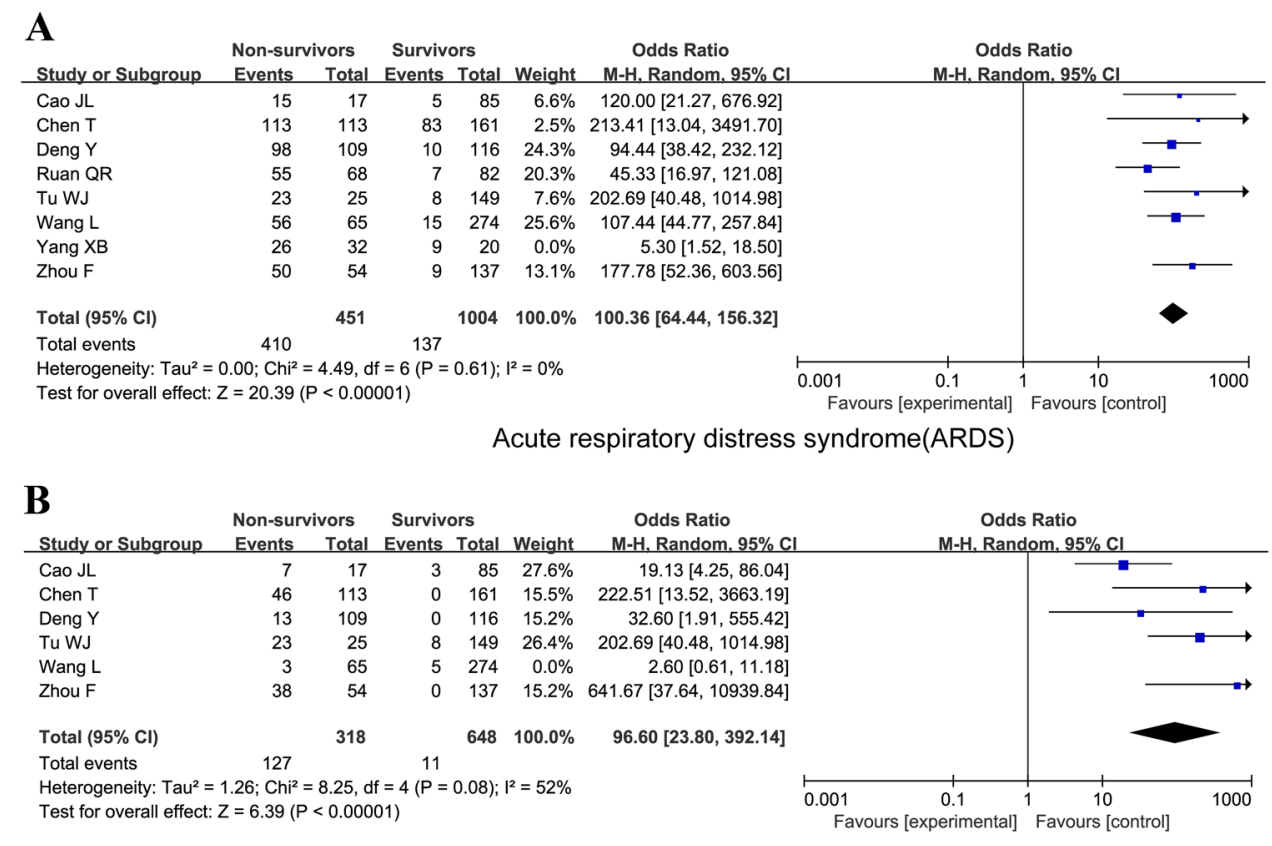

Shock anti-inflammatory, and anti-proliferation [35]. Therefore, ACE2 has a protective effect on chronic underlying diseases and ARDS [33]. But when SARS-COV-2 binds to the ACE2 receptor and enters alveolar epithelial cells through membrane fusion, the expression of ACE2 in alveolar epithelial cells is down-regulated by mechanisms such as internalization, shedding, and viral replication [36, 37]. Moreover, the COVID-19 deceased were also more likely to have a shock. The electrolyte disorder and the decrease of systemic circulation blood volume during the shock would lead to the injury of various organs in the whole body and accelerate the death of the patients [38]. We found that patients with dyspnea were more likely to develop death. Dyspnea is a sign of decreased lung function and hypoxia. Therefore, when patients have symptoms of dyspnea, clinicians must be vigilant to prevent further deterioration of the condition. Interestingly, studies had shown that high fever $\left(\geq 39^{\circ} \mathrm{C}\right)$ was positively related to the occurrence of ARDS, but negatively related to death $[39,40]$. Although our study also showed that the most common symptom of COVID-19 deceased was fever. Unfortunately, we could not clarify the inevitable relationship between the death of COVID-19 patients and the degree of fever based on the available data.

Our analysis of the biochemical results showed that some indices were abnormally elevated in the blood of COVID-19 deceased, including LDH, AST, and Scr, which indicated the injury of the myocardium, liver, and kidney. This result is consistent with the extensive distribution of SARS-CoV-2 receptors ACE2, and can also partially explain why some patients died from multiple organ failure [6]. SARS-CoV-2 can cause tissues and organs injury by direct and/or indirect action [41]. The direct injury is to infect corresponding cells by identifying the ACE2 receptor. Another important cause for multiple organ failure and even death in critical patients is the release of cytokines from the inflammatory storm, causing systemic immune injury [42]. During the early stage of the inflammatory storm, the D-dimer increases significantly, which is the result of inflammation activating plasmin. The inflammation progresses and the presence of hypoxia will result in an overall hypercoagulable state or even disseminated intravascular coagulation. The inflammatory storm is also the main cause of eventual death in many patients with advanced SARS-CoV and MERS-CoV infection $[43,44]$. In our study, we also observed a significant increase in IL-6 among COVID-19 deceased. IL-6 is a good predictor of disease severity and prognosis because it is expressed longer than other cytokines (such as TNF and IL-1) [45].

There are several limitations to our analysis and review. First, most of the included studies were cross-sectional studies with insufficient demonstration ability and lack of randomized controlled trials with optimized design. Second, our evidence was mostly from China. It would be better to include as many studies with a broad geographic scope and multiple races, to get a more comprehensive understanding of COVID-19 deceased. Third, the clinical data of patients provided by some studies were not comprehensive enough. Fourth, the actual cause of COVID-19 deceased might be obscured by unmeasured or unknown confounders. Despite our relatively large sample size, well-designed trials of high quality are needed to overcome inherent statistical bias and confirming the relationship between death and COVID-19. 


\section{Conclusion}

According to the results of the current study, the majority of COVID-19 deaths have occurred in elderly men. Typical symptoms (fever, dyspnea, dry cough, and fatigue), chronic underlying diseases (hypertension, cardiovascular disease, diabetes, and cerebrovascular disease), associated laboratory abnormalities (low platelet count, increased CRP, and LDH), and complications (ARDS and shock) are risk factors for death in COVID-19 patients. Pay close attention to these risk factors and identify critical patients early. Personalized treatment regimens are needed to enhance the efficacy and reduce the risk of COVID-19 related death.

Author contributions All the authors designed the study. Peishan Qiu, Yunjiao Zhou, and Fan Wang designed the literature search and searched the articles. Peishan Qiu, Yunjiao Zhou, Haizhou Wang, and Meng Zhang contributed to the data extraction process. All the authors analysed the data. Peishan Qiu wrote the first draft of article. All the authors revised the article and approved the final version.

Funding This work was supported by the following projects:

National Natural Science Foundation of China $(81,401,306)$ and Clinical Research Project of Guangzhou Medical University (No. 2017[160]) and Guangdong Province Science and Technology Program (No. 2016ZC0143).

National Natural Science Foundation of China (81472735); Wuhan University (2042019kf0206); National Basic Research Program of China (973program, 2015CB932600).

Availability for data and material The datasets used and/or analysed during the current study are available from the corresponding author on reasonable request.

\section{Compliance with ethical standards}

Conflict of interest On behalf of all authors, the corresponding author states that there is no conflict of interest.

Ethical approval This is a review article and no human or animal participants were recruited to this study.

Human and animal rights This is a review article. All data used were from published articles, which were following the ethical standards of the institutional review board/international ethics committee for each center and with the 1964 Helsinki Declaration and its later amendments.

Informed consent All data used in this article were from studies that had obtained the informed consent of all individual participants.

\section{References}

1. Lu H, Stratton CW, Tang YW (2020) Outbreak of pneumonia of unknown etiology in Wuhan, China: the mystery and the miracle. J Med Virol 92:401-402
2. Hui DS, IA E, Madani TA et al (2020) The continuing 2019-nCoV epidemic threat of novel coronaviruses to global health-the latest 2019 novel coronavirus outbreak in Wuhan, China. Int J Infect Dis 91:264-266

3. Huang C, Wang Y, Li X et al (2020) Clinical features of patients infected with 2019 novel coronavirus in Wuhan. China Lancet 395:497-506

4. Lu R, Zhao X, Li J et al (2020) Genomic characterisation and epidemiology of 2019 novel coronavirus: implications for virus origins and receptor binding. Lancet 395:565-574

5. Coronavirus disease 2019 (COVID-19) Situation Report-84 (https ://www.who.int/emergencies/diseases/novel-coronavirus-2019/ situation-reports/). Accessed 13 Apr 2020

6. Wang D, Hu B, Hu C et al (2020) Clinical Characteristics of 138 Hospitalized Patients With 2019 Novel Coronavirus-Infected Pneumonia in Wuhan. China. JAMA 323:1061

7. Stang A (2010) Critical evaluation of the Newcastle-Ottawa scale for the assessment of the quality of nonrandomized studies in meta-analyses. Eur J Epidemiol 25:603-605

8. Cao J, Tu WJ, Cheng W et al (2020) Clinical Features and Shortterm Outcomes of 102 Patients with Corona Virus Disease 2019 in Wuhan, China. Clin Infect Dis

9. Chen T, Wu D, Chen H et al (2020) Clinical characteristics of 113 deceased patients with coronavirus disease 2019: retrospective study. BMJ 368:m1091

10. Deng Y, Liu W, Liu K et al (2020) Clinical characteristics of fatal and recovered cases of coronavirus disease 2019 (COVID-19) in Wuhan, China: a retrospective study. Chin Med J (Engl) 11:1261

11. Du RH, Liang LR, Yang CQ et al (2020) Predictors of mortality for patients with COVID-19 pneumonia caused by SARS-CoV-2: a prospective cohort study. Eur Respir J 55:200524

12. Du Y, Tu L, Zhu P et al (2020) Clinical features of 85 fatal cases of COVID-19 from Wuhan: a retrospective observational study. Am J Respir Crit Care Med 201:1372

13. Hang Y YR, Xu Y, Gong P (2020) Clinical characteristics of 36 non-survivors with COVID-19 in Wuhan, China. medRxiv

14. Korean Society of Infectious D, Korea Centers for Disease C, Prevention (2020) Analysis on 54 mortality cases of coronavirus disease 2019 in the Republic of Korea from January 19 to March 10, 2020. J Korean Med Sci 35:e132

15. Li X, Wang L, Yan S et al (2020) Clinical characteristics of 25 death cases with COVID-19: a retrospective review of medical records in a single medical center, Wuhan. China. Int J Infect Dis $94: 128$

16. Covid-19 National Emergency Response Center E, Case Management Team KCfDC, Prevention (2020) Coronavirus disease-19: the first 7,755 cases in the Republic of Korea. Osong Public Health Res Perspect 11:85-90

17. Ruan Q, Yang K, Wang W et al (2020) Clinical predictors of mortality due to COVID-19 based on an analysis of data of 150 patients from Wuhan. China. Intensive Care Med 46:846

18. Tang N, Bai H, Chen X et al (2020) Anticoagulant treatment is associated with decreased mortality in severe coronavirus disease 2019 patients with coagulopathy. J Thromb Haemost 18:1094

19. Tu WJ, Cao J, Yu L et al (2020) Clinicolaboratory study of 25 fatal cases of COVID-19 in Wuhan. Intensive Care Med 46:1117-1120

20. Wang L, He W, Yu X et al (2020) Coronavirus disease 2019 in elderly patients: characteristics and prognostic factors based on 4-week follow-up. J Infect 80:639

21. Yang X, Yu Y, Xu J et al (2020) Clinical course and outcomes of critically ill patients with SARS-CoV-2 pneumonia in Wuhan, China: a single-centered, retrospective, observational study. Lancet Respir Med 8:475 
22. Zhou F, Yu T, Du R et al (2020) Clinical course and risk factors for mortality of adult inpatients with COVID-19 in Wuhan, China: a retrospective cohort study. Lancet 395:1054-1062

23. Badawi A, Ryoo SG (2016) Prevalence of comorbidities in the Middle East respiratory syndrome coronavirus (MERS$\mathrm{CoV})$ : a systematic review and meta-analysis. Int J Infect Dis 49:129-133

24. Channappanavar R, Fett C, Mack M et al (2017) Sex-based differences in susceptibility to severe acute respiratory syndrome coronavirus infection. J Immunol 198:4046-4053

25. Jaillon S, Berthenet K, Garlanda C (2019) Sexual dimorphism in innate immunity. Clin Rev Allergy Immunol 56:308-321

26. Gal-Oz ST, Maier B, Yoshida H et al (2019) ImmGen report: sexual dimorphism in the immune system transcriptome. Nat Commun 10:4295

27. Zhu Y, Liu C, Zhang L et al (2020) How to control the economic burden of treating cardio-cerebrovascular diseases in China? Assessment based on system of health accounts 2011. J Glob Health 10:010802

28. Mertz D, Kim TH, Johnstone J et al (2013) Populations at risk for severe or complicated influenza illness: systematic review and meta-analysis. BMJ 347:f5061

29. Odegaard JI, Chawla A (2012) Connecting type 1 and type 2 diabetes through innate immunity. Cold Spring Harb Perspect Med 2:a007724

30. Pinto BG, Oliveira AE, Singh Y et al (2020) ACE2 Expression is Increased in the Lungs of Patients with Comorbidities Associated with Severe COVID-19. J Infect Dis 222:556-563

31. Lippi G, Wong J, Henry BM (2020) Hypertension and its severity or mortality in Coronavirus Disease 2019 (COVID-19): a pooled analysis. Pol Arch Intern Med 130:304

32. Tikellis C, Bernardi S, Burns WC (2011) Angiotensin-converting enzyme 2 is a key modulator of the renin-angiotensin system in cardiovascular and renal disease. Curr Opin Nephrol Hypertens 20:62-68

33. Cheng H, Wang Y, Wang GQ (2020) Organ-protective effect of angiotensin-converting enzyme 2 and its effect on the prognosis of COVID-19. J Med Virol 92:726-730

34. Schouten LR, Helmerhorst HJ, Wagenaar GT et al (2016) Agedependent changes in the pulmonary renin-angiotensin system are associated with severity of lung injury in a model of acute lung injury in rats. Crit Care Med 44:e1226-e1235
35. Santos RA, Simoes e Silva AC, Maric C et al (2003) Angioten$\sin -(1-7)$ is an endogenous ligand for the $\mathrm{G}$ protein-coupled receptor Mas. Proc Natl Acad Sci USA 100:8258-8263

36. Verdecchia P, Cavallini C, Spanevello A et al (2020) COVID-19: ACE2centric infective disease? Hypertension 76:294

37. Glowacka I, Bertram S, Herzog P et al (2010) Differential downregulation of ACE2 by the spike proteins of severe acute respiratory syndrome coronavirus and human coronavirus NL63. J Virol 84:1198-1205

38. Zuccari S, Damiani E, Domizi R et al (2020) Changes in cytokines, haemodynamics and microcirculation in patients with sepsis/septic shock undergoing continuous renal replacement therapy and blood purification with CytoSorb. Blood Purif 49:107-113

39. Wu C, Chen X, Cai Y et al (2020) Risk factors associated with acute respiratory distress syndrome and death in patients with coronavirus disease 2019 pneumonia in Wuhan. China. JAMA Intern Med 180:934

40. Schell-Chaple HM, Puntillo KA, Matthay MA et al (2015) Body temperature and mortality in patients with acute respiratory distress syndrome. Am J Crit Care 24:15-23

41. Liu B, Li M, Zhou Z et al (2020) Can we use interleukin-6 (IL6) blockade for coronavirus disease 2019 (COVID-19)-induced cytokine release syndrome (CRS)? J Autoimmun 111:102452

42. Castrucci MR (2018) Factors affecting immune responses to the influenza vaccine. Hum Vaccin Immunother 14:637-646

43. Lew TW, Kwek TK, Tai D et al (2003) Acute respiratory distress syndrome in critically ill patients with severe acute respiratory syndrome. JAMA 290:374-380

44. Min CK, Cheon S, Ha NY et al (2016) Comparative and kinetic analysis of viral shedding and immunological responses in MERS patients representing a broad spectrum of disease severity. Sci Rep 6:25359

45. Tanaka T, Narazaki M, Kishimoto T (2016) Immunotherapeutic implications of IL-6 blockade for cytokine storm. Immunotherapy 8:959-970

Publisher's Note Springer Nature remains neutral with regard to jurisdictional claims in published maps and institutional affiliations.

\section{Affiliations}

\section{Peishan Qiu ${ }^{1,2} \cdot$ Yunjiao Zhou ${ }^{1,2} \cdot$ Fan Wang $^{1,2} \cdot$ Haizhou Wang ${ }^{1,2} \cdot$ Meng Zhang $^{1,2} \cdot$ Xingfei Pan $^{3} \cdot$ Qiu Zhao $^{1,2}$. Jing Liu ${ }^{1,2}$}

1 Department of Gastroenterology, Zhongnan Hospital of Wuhan University, No. 169, Donghu Road, Wuchang District, Wuhan 430071, Hubei Province, China

2 Hubei Clinical Center and Key Laboratory of Intestinal and Colorectal Diseases, Wuhan 430071, China
3 Department of Infectious Diseases, The Third Affiliated Hospital of Guangzhou Medical University, Guangzhou 510145, China 